archives-ouvertes

\title{
Generalized Homogenization of Linear Observers: Theory and Experiment
}

\author{
Siyuan Wang, Andrey Polyakov, Gang Zheng
}

\section{To cite this version:}

Siyuan Wang, Andrey Polyakov, Gang Zheng. Generalized Homogenization of Linear Observers: Theory and Experiment. International Journal of Robust and Nonlinear Control, Wiley, 2021, 10.1002/rnc.5771 . hal-03382805

\section{HAL Id: hal-03382805 \\ https://hal.inria.fr/hal-03382805}

Submitted on 18 Oct 2021

HAL is a multi-disciplinary open access archive for the deposit and dissemination of scientific research documents, whether they are published or not. The documents may come from teaching and research institutions in France or abroad, or from public or private research centers.
L'archive ouverte pluridisciplinaire HAL, est destinée au dépôt et à la diffusion de documents scientifiques de niveau recherche, publiés ou non, émanant des établissements d'enseignement et de recherche français ou étrangers, des laboratoires publics ou privés. 


\title{
Generalized Homogenization of Linear Observers: Theory and Experiment
}

\author{
Siyuan Wang ${ }^{1}$, Andrey Polyakov ${ }^{1,2}$, and Gang Zheng ${ }^{1,3}$ \\ ${ }^{1}$ Univ. Lille, Inria, CNRS, Centrale Lille, UMR 9189 CRIStAL, F-59000, Lille, \\ France \\ ${ }^{2}$ ITMO University, Saint-Petersburg Russia \\ ${ }^{3}$ School of Mathematics and Big Data, Foshan University, Foshan, China
}

\begin{abstract}
A procedure of upgrading a linear observer to homogeneous (nonlinear) one is proposed and validated by experiment. The nonlinear observer design is based on the the concept of a linear geometric homogeneity. The simple procedure developed to apply the homogeneous nonlinear observer is based on the parameters provided by the linear observer, which is potential for many applications to improve their performance. A saturation function is introduced to guaranteed the improvement of estimation quality. Finally the theoretical results are confirmed by the QDrone platform of Quanser ${ }^{T M}$.
\end{abstract}

\section{Introduction}

Linear observers, such as Luenberger observer 1, have been widely used in practice to estimate system's states via the information provided by sensors and actuators. In general, the structure of classical linear observers is relatively simple but with an asymptotic convergence. Two methods are adopted in the literature to boost the convergence. The first one is to use larger gain, yielding the so-called high-gain observer, which is however sensitive to the measurement noise (due to its high gain) and may result in the peaking phenomenon for the closed-loop system 2. The second method is to introduce nonlinear terms to construct a nonlinear observer with non-asymptotic convergence 3, for example sliding mode observer 4. Nevertheless, it is still a challenging problem to properly choose the gains of those nonlinear observers [4].

Recently, the homogeneous controller has been implemented to guarantee the finite-time convergence by upgrading a linear controller, which means the gains of homogeneous controller were calculated via the gains of this linear controller [5. As a dual result, this paper aims at extending the same idea to upgrade the linear observer to a homogeneous (nonlinear) one. In fact, homogeneity presents a certain symmetry of an object (e.g. a function) which might provide an alternative concept for designing observers with better precision, faster convergence and more robustness. Mathematically speaking, a symmetry of object means it is invariant with respect to a class of transformations, called dilations. For example, standard homogeneity is the symmetry of a function $f$ with respect to the 
uniform dilation $x \mapsto e^{s} x$, where $s \in \mathbb{R}$ is the scaling parameter. All the standard homogeneous function $f$ satisfies $f\left(e^{s} x\right)=e^{\mu s} f(x)$, where the constant $\mu \in \mathbb{R}$ is called homogeneity degree. The characterization of homogeneity depends on the dilation rule, such that if the dilation is changed, then a new homogeneity (generalized homogeneity ) can be generated [6], 7]. In this paper we mainly deal with the homogeneity with linear dilation 8 $x \mapsto e^{G_{\mathbf{d}} s} x$, where $x \in \mathbb{R}^{n}, s \in \mathbb{R}$ and $G_{\mathbf{d}} \in \mathbb{R}^{n \times n}$ is an anti-Hurwitz matrix 1

In the view of a larger sense 9], homogeneous (symmetric) systems include many nonlinear and all linear models of mechanics and physics. When the linearization of nonlinear system is impossible to obtain or it is too conservative, homogeneous models provide an option for the local approximation of control systems [6] 10]. Many methods applied in both linear and nonlinear control theory can be also used in design and analysis of homogeneous control system (see [6, 11, 17, 12, 13, 8, 14] and references therein). Since homogeneous system allows faster convergence [12, 15], it improves the precision and robustness of the system [10, [13.

Linear controller working with linear observer has been widely applied in many plants. Like controllers, the performance of observer might influence the performance of the closed-loop system. It can be evaluated by many quantitative indexes, which include the precision, robustness with respect to noises, etc [16, [17, 18. A well-tuned linear observer (like Luenberger or Kalman filter) can guarantee a relatively good performance in many practical cases.

In order to further improve the estimation precision, the homogeneity as a certain relaxation of linearity could provide additional tools for it. In our previous paper [5], the methodology of "upgrading" linear controllers to homogeneous (nonlinear) ones has already been developed, and it has been validated via Quanser's quadrotor platform (see the video https://youtu.be/wnSi6jj1TwE) where the set point tracking precision has been improved about $40 \%$ and the homogeneous controller has shown its better robustness than linear controller. However the observer used in [5] is still linear one, and we did not prove the global convergence since the separate principle is not valid.

This paper investigates the upgrading of linear observer to a homogeneous (nonlinear) one with finite-time convergence and better robustness with respect to noise, which enables us to prove the global finite-time convergence of the closed-loop system. The obtained homogeneous observer guarantees the further improvement of an estimation precision based on homogeneous controller and validates its efficiency on real experiments.

Notation: $\mathbb{R}$ is the set of real numbers, $\mathbb{R}_{+}=\{x \in \mathbb{R}: x>0\} ; P \succ 0$ $(\prec 0, \succeq 0, \preceq 0)$ for $P \in \mathbb{R}^{n \times n}$ means that the matrix $P$ is symmetric and positive definite (negative definite, positive semi-definite, negative semi-definite); $\lambda_{\min }(P)$ and $\lambda_{\max }(P)$ represent the minimal and maximal eigenvalue of a matrix $P=P^{\top}$; for $P \succeq 0$ the square root of $P$ is a matrix $M=P^{\frac{1}{2}}$ such that $M^{2}=P$; for $x \in \mathbb{R}^{n},\|x\|$ denotes a norm in $\mathbb{R}^{n}$, specially $\|x\|_{\mathbb{R}^{n}}$ and $\|x\|_{P}$ with $P \in \mathbb{R}^{n \times n}$ represent respectively the standard Euclidean norm and the weighted Euclidean norm in $\mathbb{R}^{n}$, i.e., $\|x\|_{\mathbb{R}^{n}}=\sqrt{x^{T} x}$ and $\|x\|_{P}=\sqrt{x^{T} P x}$.

\footnotetext{
${ }^{1} \mathrm{~A}$ matrix is said to be anti-Hurwitz if the real parts of all its eigenvalues are positive.
} 


\section{Preliminaries}

\subsection{Generalized Homogeneity}

As explained in Section 1, the homogeneity is a symmetry of an object (e.g. a function) with respect to a group of transformations, called dilations. In the general case, instead of the uniform dilation of an argument $x \mapsto$ $e^{s} x, x \in \mathbb{R}^{n}, s \in \mathbb{R}$, we can consider a non-uniform one

$$
x \mapsto \mathbf{d}(s) x, \quad x \in \mathbb{R}^{n}, s \in \mathbb{R}
$$

where $\mathbf{d}(s) \in \mathbb{R}^{n \times n}$ and $s \in \mathbb{R}$ is a parameter of the dilation. To be a dilation, the family of operators $\mathbf{d}(s)$ must satisfy some restrictions [19]: 1) $\mathbf{d}(0) x=x ; 2)\|\mathbf{d}(s) x\| \rightarrow 0$ as $s \rightarrow-\infty ; 3)\|\mathbf{d}(s) x\| \rightarrow+\infty$ as $s \rightarrow+\infty$, for $x \neq 0$.

In [7, [11, [6], the dilation $\mathbf{d}$ is suggested to be generated as a flow of a $C^{1}$ vector field $\nu: \mathbb{R}^{n} \mapsto \mathbb{R}^{n}$. Such a dilation $\mathbf{d}(s)$ is known as geometric dilation [11, 7]. In this paper we only deal with the so-called linear geometric dilations, which require the vector field $\nu$ to be linear, i.e. $\frac{d}{d s} \mathbf{d}(s)=G_{\mathbf{d}} \mathbf{d}(s)$, where $G_{\mathbf{d}} \in \mathbb{R}^{n \times n}$ is an anti-Hurwitz matrix. The matrix $G_{\mathbf{d}}$ is called a generator of the dilation. The dilation $\mathbf{d}$ in this case is given by the matrix exponential

$$
\mathbf{d}(s):=e^{G_{\mathbf{d}} s}=\Sigma_{i=0}^{\infty} \frac{s^{i} G_{\mathbf{d}}^{i}}{i !} .
$$

The dilation $\mathbf{d}$ is said to be monotone if $\mathbf{d}(s)$ is a strong contraction for any $s<0$; or strictly monotone if $\exists \beta>0$ :

$$
\|\mathbf{d}(s)\|:=\sup _{x \neq \mathbf{0}} \frac{\|\mathbf{d}(s) x\|}{\|x\|} \leq e^{\beta s}, \quad \forall s \leq 0 .
$$

Monotonicity of dilation plays an important role for the investigations of homogeneous geometrical structures in $\mathbb{R}^{n}$ as well as for the analysis of homogeneous control systems. In a finite dimensional space, any dilation is monotone and strictly monotone if a norm in $\mathbb{R}^{n}$ is properly selected.

Theorem 2.1[8] The dilation $\mathbf{d}$ is strictly monotone in $\mathbb{R}^{n}$ equipped with the weighted Euclidean norm $\|z\|=\|z\|_{P}=\sqrt{z^{\top} P z}$, where $0 \prec P=P^{\top} \in$ $\mathbb{R}^{n \times n}$ is a symmetric positive definite matrix, if and only if the following linear matrix inequality holds

$$
P G_{\mathbf{d}}+G_{\mathbf{d}}^{\top} P \succ 0, \quad P \succ 0
$$

where $G_{\mathbf{d}} \in \mathbb{R}^{n}$ is the generator of the dilation $\mathbf{d}$.

A dilation allows a new norm-topology to be introduced using the socalled canonical homogeneous norm [8]. The function $\|\cdot\|_{\mathrm{d}}: \mathbb{R}^{n} \mapsto[0,+\infty)$ defined as $\|0\|_{\mathbf{d}}=0$ and

$$
\|x\|_{\mathbf{d}}=e^{s_{x}}, \text { where } s_{x} \in \mathbb{R}:\left\|\mathbf{d}\left(-s_{x}\right) x\right\|=1,
$$

is called the canonical homogeneous norm.

Obviously, $\|\mathbf{d}(s) x\|_{\mathbf{d}}=e^{s}\|x\|_{\mathbf{d}}$ and $\|x\|_{\mathbf{d}}=\|-x\|_{\mathbf{d}}$ for any $x \in \mathbb{R}^{n}$ and any $s \in \mathbb{R}$. Notice that for $\mathbf{d}(s)=e^{s}$ the canonical homogeneous norm $\|\cdot\|$ coincides with the norm $\|\cdot\|$ on the whole $\mathbb{R}^{n}$.

The monotonicity of the dilation group guarantees that the function $\|\cdot\|_{\mathbf{d}}$ is single-valued and continuous at the origin 8 . Moreover, if we define the norm in (4) as the weighted Euclidean norm, i.e., $\|x\|=\|x\|_{P}=$ 
$\sqrt{x^{\top} P x}$ with $P \in \mathbb{R}^{n \times n}$ satisfying $(3)$, then $\|\cdot\|_{\mathbf{d}}$ is continuously differentiable outside the origin [20, and its derivative with respect to $x$ can be computed as follows

$$
\frac{\partial\|x\|_{\mathbf{d}}}{\partial x}=\|x\|_{\mathbf{d}} \frac{x^{\top} \mathbf{d}^{\top}\left(-\ln \|x\|_{\mathbf{d}}\right) P \mathbf{d}\left(-\ln \|x\|_{\mathbf{d}}\right)}{x^{\top} \mathbf{d}^{\top}\left(-\ln \|x\|_{\mathbf{d}}\right) P G_{\mathbf{d}} \mathbf{d}\left(-\ln \|x\|_{\mathbf{d}}\right) x}, \quad x \neq \mathbf{0} .
$$

It is well known (see e.g. 21]) that the weighted Euclidean norm $\|x\|_{P}=\sqrt{x^{\top} P x}$, with $P \in \mathbb{R}^{n \times n}$ being a symmetric positive definite matrix, is a Lyapunov function for any stable linear system $\dot{x}=A x, A \in$ $\mathbb{R}^{n \times n}$. It is expectable that the canonical homogeneous norm $\|x\|_{\mathrm{d}}$ might define as well a Lyapunov function for a class of stable homogeneous systems.

\subsection{Homogeneous Systems}

Homogeneity (dilation symmetry) of the vector fields is given by the following definition, which originally has been proposed by L. Euler in 18th century for the uniform dilation. The generalized homogeneity is studied in [6, 7], 10, 14.

Definition 2.1 A vector field $f: \mathbb{R}^{n} \mapsto \mathbb{R}^{n}$ is said to be $\mathbf{d}$-homogeneous of degree $\mu \in \mathbb{R}$ if

$$
f(\mathbf{d}(s) x)=e^{\mu s} \mathbf{d}(s) f(x) \quad \text { for } \quad s \in \mathbb{R}, \quad x \in \mathbb{R}^{n} .
$$

A lot of examples of $\mathbf{d}$-homogeneous nonlinear vector fields can be found in the literature. Obviously, any linear vector field $x \mapsto A x, A \in \mathbb{R}^{n \times n}$ is homogeneous of the zero degree with respect to the uniform dilation $x \mapsto$ $e^{s} x$. However, in some cases the linear vector field may be $\mathbf{d}$-homogeneous of positive or negative degree depending on the chosen dilation d. In general, the vector field $x \mapsto A x$ with $A \in \mathbb{R}^{n \times n}$ and $x \in \mathbb{R}^{n}$ is dhomogeneous of degree $\mu \in \mathbb{R}$ if and only if 8 ]

$$
A G_{\mathbf{d}}=\left(\mu I+G_{\mathbf{d}}\right) A,
$$

where $G_{\mathbf{d}} \in \mathbb{R}^{n \times n}$ is a generator of $\mathbf{d}$.

Homogeneity of a function (a vector field) is inherited by other mathematical objects induced by this function such as derivatives or solutions of differential equations. If the right hand side of the following differential equation

$$
\dot{\xi}=f(\xi), \quad t>0, f: \mathbb{R}^{n} \rightarrow \mathbb{R}^{n}
$$

is $\mathbf{d}$-homogeneous of degree $\mu$ then

$$
x_{\mathbf{d}(s) x_{0}}(t)=\mathbf{d}(s) x_{x_{0}}\left(e^{\mu s} t\right), t>0
$$

where $x_{x_{0}}(t), t>0$ denotes a solution of 8 with the initial condition $x(0)=x_{0}$ (see e.g. 8) $)$. The obtained symmetry of solution implies the fast (finite-time/fixed-time) convergence properties of the homogeneous systems dependently of the homogeneity degrees. The next proposition was originally proven for the weighted homogeneous systems in [15].

Proposition 2.1 [20] If the system [8] is $\mathbf{d}$-homogeneous of degree $\mu \in \mathbb{R}$ and its origin is locally uniformly asymptotically stable then

- for $\mu<0$ it is globally uniformly finite-time stable, i.e. there exists a d-homogeneous settling-time function $T: \mathbb{R}^{n} \mapsto[0,+\infty)$ of degree 1 , which is locally bounded and continuous at 0 , such that $x_{x_{0}}(t)=$ $0, \forall t \geq T\left(x_{0}\right)$ 
- for $\mu=0$ it is globally uniformly asymptotically stable;

- for $\mu>0$ it is globally uniformly nearly fixed-time stable, i.e. $\forall r>0$, $\exists T=T(r)>0:\left\|x_{x_{0}}(t)\right\|<r, \forall t \geq T, \forall x_{0} \in \mathbb{R}^{n}$.

This paper deals with upgrading an existing linear observer to a homogeneous one, therefore by regarding $\xi$ in (8) as the observation error and $f(\xi)=A \xi+g(C \xi)$, the objective of this paper is to seek proper function $g$, constructed from the existing Luenberger observer, such that the observation error $\xi$ can converge to zero in a finite time. Such an idea will be detailed in the next section.

\section{Homogenization of Linear Observer}

\subsection{Homogeneous State-Estimation of Linear MIMO Systems}

Let us first introduce the following linear MIMO system

$$
\dot{x}=A x+B u, \quad y=C x, \quad t>0,
$$

where $A \in \mathbb{R}^{n \times n}, B \in \mathbb{R}^{n \times m}, C \in \mathbb{R}^{k \times n}$ are system matrices, $x(t) \in \mathbb{R}^{n}$ represents state, $u(t) \in \mathbb{R}^{m}$ denotes input and $y(t) \in \mathbb{R}^{k}$ is known as measured output. Without loss of the generality, it is assumed that the matrix $C$ is of full row rank and the system (9) is observable in the sense that $\operatorname{rank} \mathcal{O}=n$ with $\mathcal{O}=\left[\begin{array}{c}C \\ \vdots \\ C A^{n-1}\end{array}\right]$. Then the following statement has been proved in [20].

Proposition 3.1 [20] Suppose that system [9] is observable in the sense that rank $\mathcal{O}=n$ with a full row rank matrix $C \in \mathbb{R}^{k \times n}$. Then there exist proper matrix $G_{0} \in \mathbb{R}^{n \times n}$ and $\mu \in \mathbb{R}$ such that

$$
A G_{0}=\left(G_{0}+I_{n}\right) A, \quad C G_{0}=\mathbf{0}
$$

and the matrix $I_{n}+\mu\left(I_{n}+G_{0}\right)$ has non-negative eigenvalues real parts. In addition, system (9) is $\mathbf{d}$-homogeneously observable of degree $\mu \in \mathbb{R}$ with the dilation $\mathbf{d}$ being generated from $G_{\mathbf{d}}=I_{n}+\mu G_{0}$.

Definition 3.1 The system (9) is said to be $\mathbf{d}$-homogeneously observable of degree $\mu \in \mathbb{R}$, if there is an observer in the form of

$$
\dot{z}=A z+B u+g(C z-y), \quad g: \mathbb{R}^{k} \rightarrow \mathbb{R}^{n}
$$

which makes the following observation error dynamics

$$
\dot{e}=A e+g(C e), \quad e=z-x
$$

uniformly globally asymptotically stable and $\mathbf{d}$-homogeneous of degree $\mu \in$ $\mathbb{R}$. For shortness, the corresponding observer 11 is called $\mathbf{d}$-homogeneous observer of degree $\mu$.

The following theorem refines Theorem 11.1 from [20, where the optimal observer gain $L \in \mathbb{R}^{n \times k}$ needs to be solved by a system of matrix inequalities. 
Theorem 3.1 With $G_{0} \in \mathbb{R}^{n \times n}$ and $\mu \in \mathbb{R}$ derived from Proposition 3.1 . let $P \in \mathbb{R}^{n \times n}, L=P^{-1} C^{T} \in \mathbb{R}^{n \times k}, \rho>0, \gamma>0$ satisfy the following system of matrix inequalities

$$
\begin{array}{r}
\left(I_{n}+\mu G_{0}\right)^{\top} P+P\left(I_{n}+\mu G_{0}\right) \succ 0, \\
P A+A^{\top} P+C^{\top} L^{\top} P+P L C+2 \rho P \prec 0, \\
P \succ \gamma^{2} C^{\top} C, \\
\rho^{2} P^{-1} \succ \Xi(\lambda) L L^{\top} \Xi^{\top}(\lambda), \quad \forall \lambda \in\left[0, \frac{1}{\gamma}\right],
\end{array}
$$

where $\Xi(\lambda)=\lambda\left(\exp \left(\ln \lambda^{\mu}\left(G_{0}+I_{n}\right)\right)-I_{n}\right)$. Then the dynamics 11 with

$$
g(\sigma)=\exp \left(\ln \|\sigma\|_{\mathbb{R}^{k}}\left(G_{0}+I_{n}\right) \mu\right) L \sigma, \quad \sigma \in \mathbb{R}^{k}
$$

is a $\mathbf{d}$-homogeneous observer of degree $\mu$ of (9) with the dilation $\mathbf{d}$ being generated from $G_{\mathbf{d}}=I_{n}+\mu G_{0}$, guaranteeing that the observation error dynamics (12) is globally uniformly asymptotically stable and $\mathbf{d}$-homogeneous of degree $\mu$.

Proof: Firstly we need to discuss the continuity of function $g$ defined in 15). The structure of function $g$ shows that $\sigma=0$ is the only one possible discontinuity point. After transforming the function $g$ in the following form

$$
g(\sigma)=\exp \left(\ln \|\sigma\|_{\mathbb{R}^{k}}\left(I_{n}+\left(G_{0}+I_{n}\right) \mu\right)\right) L \frac{\sigma}{\|\sigma\|_{\mathbb{R}^{k}}}
$$

then obviously $g(\sigma) \rightarrow \mathbf{0}$ as $\sigma \rightarrow \mathbf{0}$. If the matrix $I_{n}+\mu\left(G_{0}+I_{n}\right)$ is anti-Hurwitz, then $g$ is continuous at the point $\sigma=0$. If the eigenvalue real parts of the matrix $I_{n}+\mu\left(I_{n}+G_{0}\right)$ are non-negatives, $g$ could be discontinuous at the point $\sigma=0$, but bounded in any neighborhood of the point $\sigma=0$. In the latter case, Filippov theorem can be applied to analyze the solution of observer equation.

Secondly, we turn to prove the observation error system $\sqrt{12}$ is $\mathbf{d}-$ homogeneous of degree $\mu$. Obviously, due to Proposition 3.1, the first term $A e$ is $\mathbf{d}$-homogeneous of degree $\mu \in \mathbb{R}$. In order to show the function $g(\mathrm{Ce})$ is also d-homogeneous of degree $\mu$, let us consider the following equations

$$
C G_{0}=0 \Rightarrow C G_{\mathbf{d}}=C \Rightarrow C G_{\mathbf{d}}^{i}=C
$$

which implies $C \mathbf{d}(s)=C \exp (s)$ for $\forall s \in \mathbb{R}$. Hence, the following relation demonstrates the function $g(C e)$ is also $\mathbf{d}$-homogeneous of degree $\mu$

$$
\begin{aligned}
g(C \mathbf{d}(s) e) & =\|\exp (s) C e\|_{\mathbb{R}^{k}}^{\mu} \exp \left(\ln \|\exp (s) C e\|_{\mathbb{R}^{k}}^{\mu} G_{0}\right) L C \exp (s) e \\
& =\exp ((\mu+1) s) \exp \left(\mu s G_{0}\right) g(C e)=\exp (\mu s) \mathbf{d}(s) g(C e)
\end{aligned}
$$

Thus we proved that $\sqrt{12}$ is $\mathbf{d}$-homogeneous of degree $\mu \in \mathbb{R}$.

In order to prove the observation error dynamics 12 is globally uniformly asymptotically stable, let us consider the canonical homogeneous norm $\|e\|_{\mathbf{d}}$ which is defined based on the weighted Euclidean norm $\|e\|=$ $\|e\|_{P}=\sqrt{e^{\top} P e}$ and is smooth on $\mathbb{R}^{n} \backslash\{0\}$. Since canonical norm $\|e\|_{\mathbf{d}}$ is positive definite and continuously differentiable, we can then choose it as the Lyapunov function to study the stability of 12 .

The first inequality of 13 results in the dilation $\mathbf{d}$ is strictly monotone [8], which is important to prove the stability of error system. Indeed, using 
the system of matrix inequalities (13) and the formula (5), we drive the derivative of $\|e\|_{\mathbf{d}}$

$$
\begin{aligned}
\frac{d}{d t}\|e\|_{\mathbf{d}} & =\|e\|_{\mathbf{d}} \frac{e^{\top} \mathbf{d}^{\top}\left(-\ln \|e\|_{\mathbf{d}}\right) P \mathbf{d}\left(-\ln \|e\|_{\mathbf{d}}\right)(A e+g(C e))}{e^{\top} \mathbf{d}^{\top}\left(-\ln \|e\|_{\mathbf{d}}\right) P G_{\mathbf{d}} \mathbf{d}\left(-\ln \|e\|_{\mathbf{d}}\right) e} \\
& =\|e\|_{\mathbf{d}}^{1+\mu} \frac{e^{\top} \mathbf{d}^{\top}\left(-\ln \|e\|_{\mathbf{d}}\right)\left[P A \mathbf{d}\left(-\ln \|e\|_{\mathbf{d}}\right) e+P g\left(C \mathbf{d}\left(-\ln \|e\|_{\mathbf{d}}\right) e\right)\right]}{e^{\top} \mathbf{d}\left(-\ln \|e\|_{\mathbf{d}}\right) P G_{\mathbf{d}} \mathbf{d}\left(-\ln \|e\|_{\mathbf{d}}\right) e} \\
& \leq\|e\|_{\mathbf{d}}^{1+\mu} \frac{-\rho-v^{\top} P L C v+\|C v\|_{\mathbb{R}_{k}}^{\mu} v^{\top} P \exp \left(\ln \|C v\|_{\mathbb{R}_{k}}^{\mu} G_{0}\right) L C v}{v^{\top} G_{\mathbf{d}} P v} \\
& =\|e\|_{\mathbf{d}}^{1+\mu} \frac{-\rho+v^{\top} P\left[\|C v\|_{\mathbb{R}^{k}}^{\mu} \exp \left(\ln \|C v\|_{\mathbb{R}_{k}}^{\mu} G_{0}\right)-I_{n}\right] L C v}{v^{\top} P G_{\mathbf{d}} v}
\end{aligned}
$$

where $v=\mathbf{d}\left(-\ln \|e\|_{\mathbf{d}}\right) e$ belongs to the unit sphere, i.e. $v^{\top} P v=1$. According to the first LMI of the system (13), the following condition holds:

$0<v^{\top} P G_{\mathbf{d}} v=0.5 v^{\top}\left(P G_{\mathbf{d}}+G_{\mathbf{d}}^{\top} P\right) v \leq 0.5 \lambda_{\max }\left(P^{-\frac{1}{2}} G_{\mathbf{d}}^{\top} P^{\frac{1}{2}}+P^{\frac{1}{2}} G_{\mathbf{d}} P^{-\frac{1}{2}}\right)$

Since we have

$$
q^{\top} L C v \leq\|L q\|_{\mathbb{R}^{k}}\|C v\|_{\mathbb{R}^{k}}, \quad \forall q \in \mathbb{R}^{n}
$$

then denote $\lambda:=\|C v\|_{\mathbb{R}^{k}}$, we derive

$$
v^{\top} P\left[\lambda^{\mu} \exp \left(\ln \lambda^{\mu} G_{0}\right)-I_{n}\right] L C v \leq\left\|L^{\top}\left[\lambda^{\mu} \exp \left(\ln \lambda^{\mu} G_{0}^{\top}\right)-I_{n}\right] P v\right\|_{\mathbb{R}^{k}} \lambda .
$$

Therefore the inequality $\left\|L^{\top}\left[\lambda^{\mu} \exp \left(\ln \lambda^{\mu} G_{0}^{\top}\right)-I_{n}\right] P v\right\|_{\mathbb{R}_{k}} \lambda<\rho$ can be written in the following form

$$
\left.P\left[\lambda^{\mu} \exp \left(\ln \lambda^{\mu} G_{0}\right)-I_{n}\right)\right] L L^{\top}\left[\lambda^{\mu} \exp \left(\ln \lambda^{\mu} G_{0}^{\top}\right)-I_{n}\right] P \prec \frac{\rho^{2} P}{\lambda^{2}} .
$$

or, equivalently,

$$
\Xi(\lambda) L L^{\top} \Xi(\lambda) \prec \rho^{2} P^{-1} .
$$

In addition, the matrix inequality $P \succ \gamma C^{\top} C$ implies $\lambda \in[0,1 / \gamma]$.

Consequently, if 13 and (14) are satisfied, then $\exists c>0$ such that

$$
\frac{d}{d t}\|e(t)\|_{\mathbf{d}}<-c\|e(t)\|_{\mathbf{d}}^{1+\mu}
$$

which implies that the observation error dynamics 12 is globally uniformly asymptotically stable.

Finally, we proved that 11) is a $\mathbf{d}$-homogeneous observer of degree $\mu$ for the linear system $(9)$.

Remark 3.1 Since $\sup _{\lambda \in[0,1 / \gamma]}\|\Xi(\lambda)\| \rightarrow 0$ as $\mu \rightarrow 0$ (see Proposition 11.1 in [20]), the system of matrix inequalities (13) and (14) is always feasible if $\mu$ is sufficiently small. In addition, by fixing the value $\lambda \in\left[0, \frac{1}{\gamma}\right]$, (13) and 14 become a system of LMIs, which can be solved using any appropriate mathematical software.

Remark 3.2 The main difference between this paper and reference [20] is that this paper indicates the relation between the linear Luenberger observer with linear constant matrix gain $L$ and homogeneous observer with nonlinear function $g(\mathrm{Ce})$ depending on $L$, which makes the homogeneous observer easy to be implemented in the practice by the engineer. This relation can be used to significantly improve the performance of linear observer, which is supported by the experiments in the Section 4. 


\subsection{From a linear observer to a homogeneous one}

Notice that for $\mu=0$ the homogeneous observer 111, 15 becomes the Luenberger one with

$$
g(\sigma)=L \sigma, \quad \sigma=C e
$$

and the system of matrix inequalities $13,(14)$ is simplified to be

$$
P A+A^{\top} P+2 \rho P+P L C+C^{\top} L^{\top} P \prec 0, \quad P \succ 0, \quad \rho \succ 0
$$

It is well-known 22 that the feasibility of the latter inequality is the sufficient and necessary condition for the exponential stability of observation error dynamics 12 (with the decay rate $\rho>0$ ).

In order to guarantee that the homogeneous observer performance is always better than linear observer in practice, we introduce a saturation function sat $a, b: \mathbb{R}_{+} \mapsto \mathbb{R}_{+}$as follows

$$
\operatorname{sat}_{a, b}(\eta)=\left\{\begin{array}{llc}
b & \text { if } & \eta \geq b, \\
\eta & \text { if } & a<\eta<b, \\
a & \text { if } & \eta<a,
\end{array} \quad \eta \in \mathbb{R}_{+} .\right.
$$

A new function $g(\sigma)$ with a saturation function is given by

$$
g_{a, b}(\sigma)=\operatorname{sat}_{a, b}\left(\|\sigma\|_{\mathbb{R}_{k}}^{\mu}\right) \exp \left(\ln \operatorname{sat}_{a, b}\left(\|\sigma\|_{\mathbb{R}^{k}}^{\mu}\right) G_{0}\right) L \sigma,
$$

where $G_{0}$ and $L$ are defined in Theorem 3.1

From 19 we conclude that $g_{1,1}(\sigma)=L \sigma$ and

$$
g_{0,+\infty}(\sigma)=\|\sigma\|_{\mathbb{R}_{k}}^{\mu} \exp \left(\ln \|\sigma\|_{\mathbb{R}^{k}}^{\mu} G_{0}\right) L \sigma .
$$

In other words, the pair $a \in(0,1]$ and $b \in[1,+\infty)$ parameterize a family of nonlinear observers which has the Luenberger observer and homogeneous observer as the limit cases. This motivates the research for an "upgrading" of the Luenberger observer to the homogeneous one.

Assume that we have the Luenberger observer of the following form

$$
\dot{z}=A z+B u+L_{l i n}(C z-y)
$$

such that the observation error dynamics

$$
\dot{e}=A e+L_{l i n} \sigma, \quad \sigma=C e
$$

is already designed. In order to apply the method proposed in Theorem 3.1 the following algorithm can be applied:

1) Take the gain $L_{l i n} \in \mathbb{R}^{n \times k}$ of the existing Luenberger observer and select the parameters $G_{0} \in \mathbb{R}^{n \times n}$ and $\mu \in \mathbb{R}$ such that the system of matrix inequalities (13) and (14) is feasible ${ }^{2}$ with respect to $\gamma>0$, $\rho>0$ and $P \succ 0$.

2) Replace the gain $L$ of 20 by $L_{l i n}$.

3) Select $a=b=1$ which means we start from a linear observer.

4) Increase $b>1$ and decrease $a<1$ if it can improve an estimation precision or the quality of the whole control system, since the estimation precision cannot be evaluated directly.

\footnotetext{
${ }^{2}$ Computational procedures for solving the system of nonlinear matrix inequalities of the form [13, 14 are developed in [14 for a linear dilations with diagonal matrix $G_{\mathbf{d}}$.
} 
Theoretically, the improvement of control performance (for example: faster transition) follows from Proposition 2.1 and Theorem 3.1. However, their proofs are based on the mathematical model, and all mathematical models are only the approximation of real system. In practice, a serious degradation of control performance may happen as well due to this reason. The objective of introducing and tuning parameters $a$ and $b$ is to solve the above problem and to guarantee that this homogeneous observer always has a better quality than linear Luenberger one. At least, it will be never worse than Luenberger observer which prevents the possible degradation of control performance while upgrading the linear observer. In Section 4 we will illustrate the experiment results of the proposed scheme via quadrotor platform.

Notice that if the gains of linear observer have already been optimally adjusted, then the improvement based on homogeneous observer can not be huge and in this case the value of parameters $a$ and $b$ could be close 1 .

\section{Upgrade a linear filter for QDrone of Quanser $^{T M}$}

In this section, we will show how to apply the result presented in Section 3 to realize the control of quadrotor.

\subsection{Quanser platform}

Quanser QDrone ${ }^{T M}$ is a quadrotor with a powerful chip on board. The hardware parameters of quadrotor are following $m=1.07 \mathrm{~kg}$, the gravity $g=9.8 \mathrm{~m} / \mathrm{s}^{2}$, the motor distance $L_{\text {roll }}=0.2136 \mathrm{~m}, L_{\text {pitch }}=0.1758 \mathrm{~m}$, the roll inertia $I_{x x}=6.85 \times 10^{-3} \mathrm{kgm}^{2}$, the pitch inertia $I_{y y}=6.62 \times$ $10^{-3} \mathrm{kgm}^{2}$, the yaw inertia $I_{z z}=1.29 \times 10^{-2} \mathrm{kgm}^{2}$, the thrust coefficient $k=1.93 \times 10^{-8} \frac{N}{R P M^{2}}$ and the drag coefficient $c=0.26 \times 10^{-9} \frac{N m}{R P M^{2}}$.

The QDrone platform used in this paper includes two types of sensors: External OptiTrack and on-board IMU that measure the system state values in different frames with different sampling frequencies.

1. External OptiTrack: The OptiTrack system equips ultra-red camera to measure the position and attitude of quadrotor with a maximum $100 \mathrm{~Hz}$ sampling frequency in real-time (sampling frequency depends on the number of quadrotor localized: in our case only 1 quadrotor is localized). It can provide the following state information in Earth frame

$$
{ }^{E}[x, y, z, \phi, \theta, \psi]
$$

where $x, y, z$ denote the position and $\phi, \theta, \psi$ represent the Euler angle roll, pitch and yaw, respectively.

2. On-board IMU: The on-board IMU sensor including accelerometer, gyroscope, magnetometer and barometer, works with a maximum $1000 \mathrm{~Hz}$ sampling frequency. It can provide the following measurements

$$
{ }^{B}\left[\dot{\phi}, \dot{\theta}, \dot{\psi}, a_{x}, a_{y}, a_{z}, T_{x}, T_{y}, T_{z}, P_{g}\right]
$$

where $\dot{\phi}, \dot{\theta}, \dot{\psi}$ are the angular velocities around $x, y, z$ axis respectively, $a_{x}, a_{y}, a_{z}$ denote the associated acceleration along each axis, $T_{x}, T_{y}, T_{z}$ represent magnetism, and $P_{g}$ represents the air pressure. All the values measured by on-board IMU are in body frame. 
It is worth noting that these two types of sensors cannot measure the velocity $(\dot{x}, \dot{y}, \dot{z})$ or provide a synchronized data, which are problems for controller design. The next subsection will show how Quanser overcomes these two problems by linear filter.

\subsection{Linear filter of Quanser}

Concerning the controller design problem for quadrotor, considerable methods are proposed in the literature, such as $\mathrm{H}_{\infty}$, Backstepping, MPC, Sliding mode, and so on. For QDrone platform, Quanser realized 4 independent PID controllers for the regulation of $x, y, z$ and $\psi$, respectively. Recently, we presented an efficient homogeneous PID controller [5], by upgrading the Quanser's PID controller, which shows a substantial improvement of the control performance. However, two important problems need to be solved before applying those mentioned control methods

1. Unavailable information: OptiTrack and IMU cannot provide the velocity information $(\dot{x}, \dot{y}, \dot{z})$, which is necessary for the control method mentioned above. How to compute the velocity is the first problem to be overcome.

2. Asynchronous sampling frequency: The measurement of IMU has a higher sampling frequency than OptiTrack. How to combine the high frequency acceleration data with the low frequency position data to estimate the velocity is the second issue.

To solve the above two problems, Quanser proposed the following two filters to obtain a better estimation of velocity $(\dot{x}, \dot{y}, \dot{z})$

1. Differentiation: This filter is to solve the problem of computing derivatives. Precisely, the following transfer function

$$
H_{\text {diff }}(s)=\frac{2500 s}{s^{2}+100 s+2500}
$$

was used to estimate the derivative $\dot{\hat{p}}$ of the input signal $p(t)$, i.e.

$$
s \hat{p}(s)=H_{\text {diff }}(s) p(s)
$$

2. Data fusion: To overcome the second problem, Quanser designed another filter to make the data fusion which merges the reading from IMU (noted as $a_{i m u}$ ) and the estimated derivative (via the filter $H_{\text {diff }}$ of (23)) of the reading from OptiTrack (noted as $p_{\text {cam }}$ ). Consider these datum with different sampling frequencies as input signal, the following two transfer functions

$$
\begin{aligned}
H_{\text {high }}(s) & =\frac{s}{s^{2}+4 s+0.1} \\
H_{\text {low }}(s) & =\frac{4 s+0.1}{s^{2}+4 s+0.1}
\end{aligned}
$$

are applied to make the data fusion. Precisely, with OptiTrack reading $p_{\text {cam }}(t)$, its derivative $\dot{\hat{p}}_{c a m}(t)$ is estimated by

$$
s \hat{p}_{\text {cam }}(s)=H_{\text {diff }}(s) p_{\text {cam }}(s)
$$

Due to the fact that $\dot{\hat{p}}_{\text {cam }}(t)$ is low frequency data $(100 H z)$ while the on-board IMU is high frequency data $(1000 \mathrm{~Hz})$, a further improvement on the estimation of $\dot{\hat{p}}_{c a m}(t)$ by using both datum from IMU and OptiTrack can be realized by

$$
s \hat{p}_{\text {fus }}(s)=H_{\text {low }}(s) s \hat{p}_{\text {cam }}(s)+H_{\text {high }}(s) a_{i m u}(s)
$$


where $\hat{p}_{f u s}$ is the signal synchronized.

In summary, a better estimation is implemented by merging the derivative of low frequency reading of OptiTrack $p_{\text {cam }}(t)$ and high frequency reading of IMU $a_{i m u}(t)$, which can be presented as follows

$$
s \hat{p}_{f u s}(s)=H_{Q_{1}}(s) p_{\text {cam }}(s)+H_{Q_{2}}(s) a_{i m u}(s)
$$

with

$$
H_{Q_{1}}(s)=H_{\text {low }}(s) H_{\text {diff }}(s)=\frac{2500(4 s+0.1) s}{\left(s^{2}+4 s+0.1\right)\left(s^{2}+100 s+2500\right)}
$$

and

$$
H_{Q_{2}}(s)=H_{\text {high }}(s)=\frac{s}{s^{2}+4 s+0.1}
$$

It is worth noting that the position reading of OptiTrack $(x, y, z)$ and the acceleration reading of IMU $\left(a_{x}, a_{y}, a_{z}\right)$ need to be transformed into the same frame before merging them by 27). Finally it is the estimated linear velocity $(\dot{\hat{x}}, \dot{\hat{y}}, \dot{\hat{z}})$ that is used to implement the controller.

Furthermore the adopted linear filters by Quanser are relatively simple to be implemented. As to present in the next section, an upgrading of linear filter based on Quanser's parameters of linear filters, provides a better estimation precision and robustness.

\subsection{Homogenization of Quanser's filters}

In the previous subsection, we explained how Quanser designs two filters to obtain the derivative estimation from two asynchronous readings of OptiTrack and IMU. In this subsection, we detail how to apply the theoretical result of Section 3 to Quanser's filters. To do this, two steps need to be effectuated:

- Step 1: Find a linear system (LTI) which is able to estimate the linear velocity $(\dot{x}, \dot{y}, \dot{z})$ by using both position and acceleration measurements, that is the same objective as the two filters proposed by Quanser;

- Step 2: Using the theoretical result of Section 3 to upgrade the LTI to a homogeneous one, which means the homogeneous observer is based on the parameters of LTI.

The following details how to realize the above two steps.

Step 1: For the sake of simplicity, the following explains how to estimate the linear velocity along $x$-axis by using the $x$-axis position of OptiTrack reading $p_{c a m_{x}}$ and $x$-axis acceleration of IMU reading $a_{i m u_{x}}$. The same scheme is applied to estimate the linear velocity of other axes.

Consider quadrotor as a rigid body system, Newton's second law of motion leads to the quadrotor dynamics along $x$-axis as follows

$$
\begin{aligned}
& \dot{p}_{x}=v_{x} \\
& \dot{v}_{x}=a_{x}
\end{aligned}
$$

where $p_{x}$ represents the $x$-position of the mass center of the quadrotor, $v_{x}=\dot{x}$ is the velocity along $x$-axis and $a_{x}$ corresponds the acceleration associated with $x$-axis. Suppose now we have the two asynchronous readings $p_{c a m_{x}}$ and $a_{i m u_{x}}$, which have the following relations with $p_{x}$ and $a_{x}$

$$
\begin{aligned}
& p_{x}=p_{\text {cam }_{x}}-\omega(t) \\
& a_{x}=a_{i m u_{x}}+d(t)
\end{aligned}
$$


where $\omega(t)$ represents the position error along $x$-axis between the measurement of OptiTrack and the real position $p_{x}$, and $d(t)$ represents the acceleration error along $x$-axis between the measurement of IMU and the real acceleration $a_{x}$. In general, $d(t)$ can be approximated by a high-order polynomial function of $t$, however in our study $d(t)$ is assumed to be constant, i.e. $\dot{d}=0$. Hence, the dynamic model 30 can be re-written into the following LTI model with noisy output

$$
\begin{aligned}
\dot{X} & =A X+B u=A X+B a_{i m u_{x}} \\
Y & =C X+\omega=p_{\text {cam }_{x}}
\end{aligned}
$$

where $X=\left[p_{x}, v_{x}, d\right]^{T}, A=\left[\begin{array}{lll}0 & 1 & 0 \\ 0 & 0 & 1 \\ 0 & 0 & 0\end{array}\right], B=[0,1,0]^{T}, C=[1,0,0]$, and $u=a_{i m u_{x}}$

Obviously (31) is observable by checking the rank condition. Hence we can design the following Luenberger observer

$$
\dot{\hat{X}}=A \hat{X}+B a_{i m u_{x}}+L\left(p_{c a m_{x}}-C \hat{X}\right)
$$

and it is clear to see that $\hat{X}$ converges to a neighborhood of $X$ (depending on the bound of the noise $\omega$ ), if the gain $L=\left[l_{1}, l_{2}, l_{3}\right]^{T}$ makes $A-L C$ be Hurwitz.

Obviously, a more reasonable choice of $L$ is to approximate these two transfer functions $H_{Q_{1}}$ and $H_{Q_{2}}$ defined in (28) and (29), since Quanser spends considerable time to find the optimal parameters of these two transfer functions. To this aim, we apply the Laplace transformation to (32) and then a straightforward calculation yields the following relation

$$
\hat{X}_{2}(s)=H_{L_{1}}(s) p_{\text {cam }_{x}}+H_{L_{2}}(s) a_{i m u_{x}}
$$

where

$$
H_{L_{1}}(s)=\frac{s\left(l_{2} s+l_{3}\right)}{s^{3}+l_{1} s^{2}+l_{2} s+l_{3}}
$$

and

$$
H_{L_{2}}(s)=\frac{\left(s+l_{1}\right) s}{s^{3}+l_{1} s^{2}+l_{2} s+l_{3}}
$$

Hence, by comparing the above two transfer functions $H_{L_{1}}$ and $H_{L_{2}}$ with $H_{Q_{1}}$ and $H_{Q_{2}}$ defined in 28) and (29), the following value of $L=$ $\left[l_{1}, l_{2}, l_{3}\right]^{\top}$ with

$$
l_{1}=30, \quad l_{2}=4 l_{1}=120, \quad l_{3}=0.1 l_{1}=3
$$

are selected for the purpose of well fitting the bode diagrams of $H_{L_{1}}$ and $H_{L_{2}}$ to those of $H_{Q_{1}}$ and $H_{Q_{2}}$, such that the designed Luenberger observer processes the similar performance as Quanser's filters.

With the chosen parameter $L$, Fig. 1 depicts the bode diagrams of Quanser's transfer functions and those of $H_{L_{1}}$ and $H_{L_{2}}$ derived from the deduced Luenberger observer. In the left of Fig. 1 the frequency response of $H_{Q_{1}}$ defined in (28) is compared with that of $H_{L_{1}}$ defined in (34), and we can see that in the low frequency band $0-100 H z$ (the main operational frequency band for quadrotor) the transfer function $H_{L_{1}}$ has a similar magnitude as that of $H_{Q_{1}}$, but with a smaller phase shift than $H_{Q_{1}}$. Meanwhile, as depicted in the right of Fig. 11. the frequency response of $H_{L_{1}}$ matches exactly that of $H_{Q_{1}}$ for the whole frequency domain. Those comparisons prove that the deduced Luenberger observer 32 can 

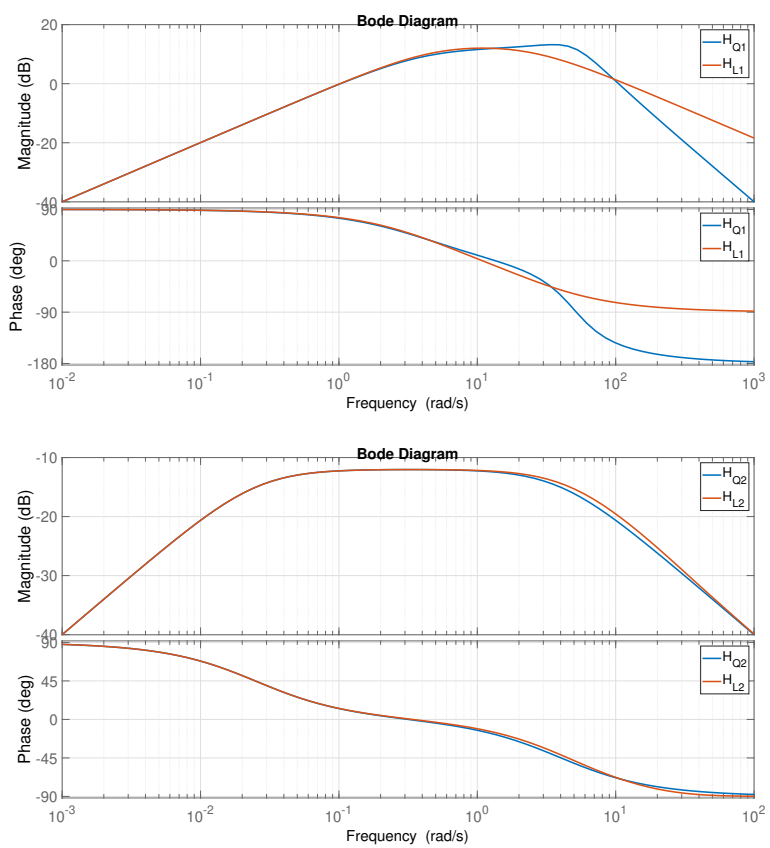

Figure 1: Bode diagram of transfer functions. Left: $H_{Q_{1}}$ and $H_{L_{1}}$; Right: $H_{Q_{2}}$ and $H_{L_{2}}$

precisely approximate Quanser's filters in the main operational frequency band for quadrotor.

Step 2: Based on the Luenberger observer proposed in 32 and its parameters (36), we can design the following homogeneous observer proposed in Section 3

$$
\dot{\hat{X}}=A \hat{X}+B a_{i m u_{x}}+g\left(p_{\text {cam }_{x}}-C \hat{X}\right)
$$

where the function $g$ defined in 20 is of the following form

$$
g\left(p_{\text {cam }_{x}}-C \hat{X}\right)=\operatorname{sat}_{a, b}\left(\|C e\|_{\mathbb{R}_{k}}^{\mu}\right) \exp \left(\ln \operatorname{sat}_{a, b}\left(\|C e\|_{\mathbb{R}^{k}}^{\mu}\right) G_{0}\right) L C e
$$

with $e=X-\hat{X}$ and $G_{0}=\left[\begin{array}{lll}0 & 0 & 0 \\ 0 & 1 & 0 \\ 0 & 0 & 1\end{array}\right], L=\left[l_{1}, l_{2}, l_{3}\right]^{T}$ is given in $[36$, and the parameters $a, b, \mu$, detailed in Section 3 , are given by the following values in our experiments: $\mu=-0.25, x$ and $y$ direction: $a=0.1, b=$ 2, $z$ direction: $a=0.25, b=1$. Please notice that if there is already a Luenberger observer in the original system, we can upgrade it to a homogeneous one directly by its parameters.

\subsection{Experiment results}

In this part, two experimental results will be presented. The first one is using Quanser's filters and homogeneous controller proposed in [5]. The second one is based on the homogeneous observer (37) and the same homogeneous controller. The comparison of these two experimental results illustrates the further improvement by homogeneous observer. The experiment is based on the set-points tracking which are defined in Earth frame: 
$[x, y, z, \psi]=[0,0,0,0] \rightarrow[0,0,0.2,0] \rightarrow\left[0.5,0,0.2, \frac{\pi}{3}\right] \rightarrow\left[0.5,0.5,0.2, \frac{\pi}{3}\right] \rightarrow$ $[-0.5,-0.5,0.8,0] \rightarrow[0,0,0.8,0] \rightarrow[0,0,0.018,0]$.

Fig. 2 shows the position stabilization trajectory of $x, y, z$ and $\psi$ respectively (experimental video, please follow https://youtu.be/4cwXG1k7Ojo). It is clear to see that the nonlinear homogeneous observer proposed in this paper provides the system a further improvement on precision, response and robustness.

Table 1: Mean value of stabilization error

\begin{tabular}{|c|c|c|c|}
\hline$L_{2}$ Error $(\mathrm{m})$ & $\mathrm{QF}+\mathrm{HC}$ & $\mathrm{HF}+\mathrm{HC}$ & Improvement \\
\hline \hline$\|$ error $_{x} \|_{L_{2}}$ & 0.0851 & 0.0760 & $10.69 \%$ \\
\hline$\|$ error $_{y} \|_{L_{2}}$ & 0.0491 & 0.0248 & $49.49 \%$ \\
\hline |error $_{z} \|_{L_{2}}$ & 0.0166 & 0.0089 & $46.39 \%$ \\
\hline$\|$ error $_{\psi} \|_{L_{2}}$ & 0.0426 & 0.0283 & $33.57 \%$ \\
\hline
\end{tabular}

The least square stabilization errors ( $L_{2}$-error) between Quanser's filter (QF) with homogeneous controller (HC) and homogeneous filter (HF) with homogeneous controller during the steady state are compared in Table 1 In both experiments, the steady state of $x, y, \psi$ and $z$ is defined from 4 and 2 sec respectively after the $z$ reference switches to $0.8 \mathrm{~m}$ and ends at the instant that the $z$ reference switches to $0.018 \mathrm{~m}$. Obviously the significant improvement of the $L_{2}$-error in $x$ and $z$ is more than $45 \%$. Since the movement of $\psi$ is coupled with other direction movements in practice, when $x, y$ and $z$ converge faster with a higher precision, the precision of $\psi$ has been improved as well which coincides with the result in Table 1 Please notice that, compared with the result of [5, Table 11 demonstrates an additional improvement by using the homogeneous observer with homogeneous controller.

\section{Conclusion}

In this paper, a simple method about how to "upgrade" a linear observer to a nonlinear homogeneous one is developed. Since the linear observer is widely applied in practice, and its parameters are well tuned by the manufacturer, the proposed method can be easily use to upgrade the existing linear observer to a homogeneous one, by using the same parameters from linear observer, and providing better performance. Such a method scales the linear parameters in a generalized homogeneous way that depends on the norm of the available estimation error $C z-y$. The saturation function has been introduced in the homogeneous observer in order to guarantee that the homogeneous observer is always better than linear one. In addition, the proposed homogeneous observer has been proved to have a faster convergence and better robustness than linear ones. Finally, the theoretical results have been validated by the real experiments on quadrotor platform ( QDrone of Quanser ${ }^{T M}$ ). The experimental results show that the generalized homogeneous observer upgrading from linear one improves the precision and robustness of original quadrotor system. 

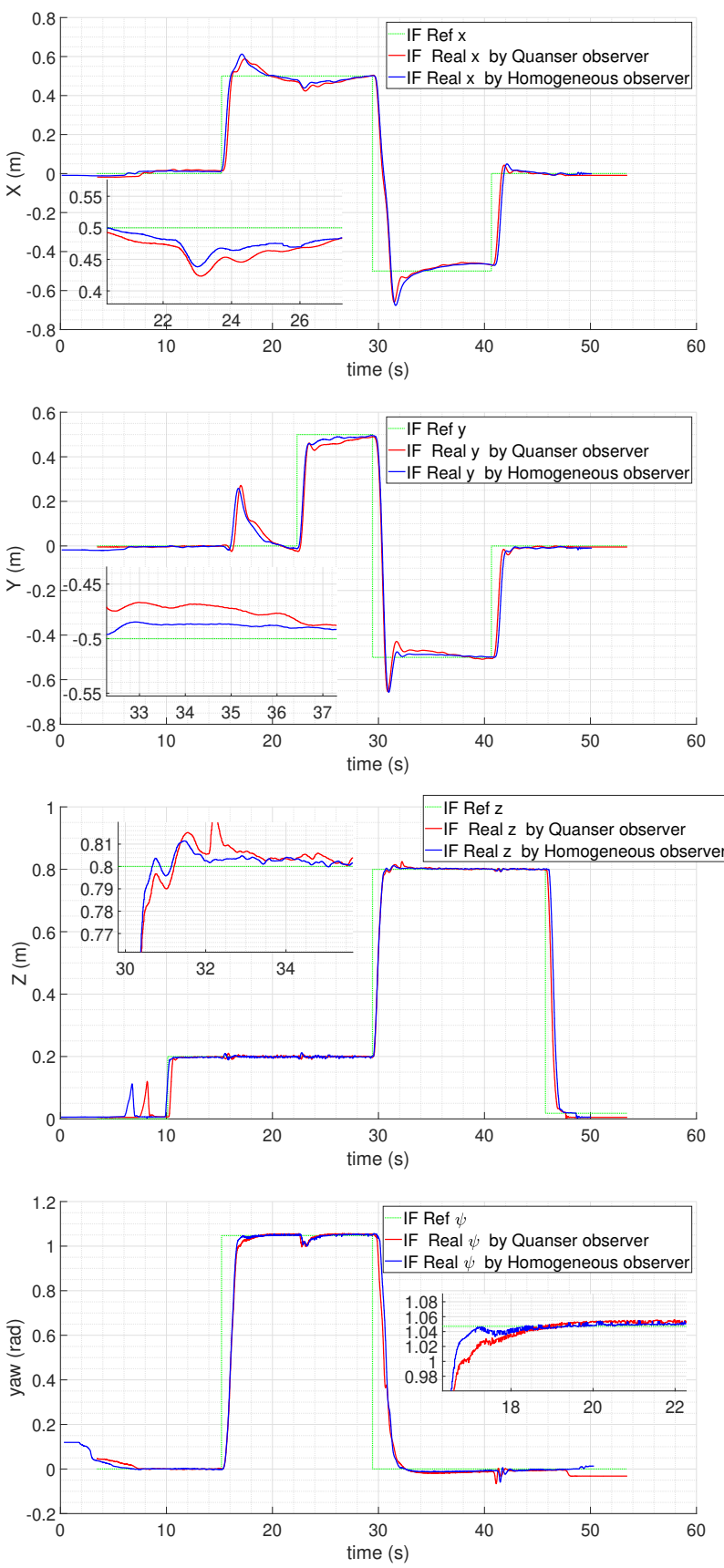

Figure 2: Quadrotor position stabilization comparisons in $x, y, z$ and $\psi$

\section{Acknowledgments}

The authors acknowledge the support of CSC Grant, CPER DATA and ANR DIGISLID, ANR-18-CE40-0008. The second author also acknowledges the support of National Natural Science Foundation of China (Grant 
No. 62050410352), the Government of Russian Federation (Grant 08-08) and the Ministry of Science and Higher Education of Russian Federation, goszadanie no. 2019-0898. The third author acknowledges the support of the National Natural Science Foundation of China (Grant No. 62073081), by the Project of Department of Education of Guangdong Province (Grant No. 2019KZDXM037), by the fund of Guangdong-Hong Kong-Macao Joint Laboratory for Intelligent Micro-Nano Optoelectronic Technology (No. 2020B1212030010).

\section{Data Availability Statement}

The data that support the findings of this study are available from the corresponding author upon reasonable request.

\section{References}

[1] Luenberger D. Observing the State of a Linear System. IEEE Transactions on Military Electronics 1964; 8(2): 74-80.

[2] Ahrens JH, Khalil HK. High-gain observers in the presence of measurement noise: A switched-gain approach. Automatica 2009; 45(4): 936-943.

[3] Davila J, Fridman L, Levant A. Second-order sliding-mode observer for mechanical systems. IEEE transactions on automatic control 2005; 50(11): 1785-1789.

[4] Utkin V, Lee H. Chattering problem in sliding mode control systems. In: IEEE International Workshop on Variable Structure Systems. ; 2006: 346-350.

[5] Wang S, Polyakov A, Zheng G. Generalized homogenization of linear controllers: Theory and experiment. International Journal of Robust and Nonlinear Control 2020.

[6] Zubov V. On systems of ordinary differential equations with generalized homogenous right-hand sides. Izvestia vuzov. Mathematica (in Russian). 1958; 1: 80-88.

[7] Rosier L. Etude de quelques Problèmes de Stabilization. PhD Thesis, Ecole Normale Superieure de Cachan (France) 1993.

[8] Polyakov A. Sliding Mode Control Design Using Canonical Homogeneous Norm. International Journal of Robust and Nonlinear Control 2018; 29(3): 682-701. doi: 10.1002/rnc.4058

[9] Orlov Y. Finite Time Stability and Robust Control Synthesis of Uncertain Switched Systems. SIAM Journal of Control and Optimization 2005; 43(4): 1253-1271.

[10] Andrieu V, Praly L, Astolfi A. Homogeneous Approximation, Recursive Observer Design and Output Feedback. SIAM Journal of Control and Optimization 2008; 47(4): 1814-1850.

[11] Kawski M. Geometric Homogeneity And Stabilization. In: Proceedings IFAC Nonlinear Control Symposium. ; 1995; Lake Tahoe, CA: 164-169.

[12] Bhat SP, Bernstein DS. Geometric homogeneity with applications to finite-time stability. Mathematics of Control, Signals and Systems 2005; 17: 101-127. 
[13] Bernuau E, Efimov D, Perruquetti W, Polyakov A. On homogeneity and its application in sliding mode control. Journal of The Franklin Institute 2014; 351(4): 1866-1901.

[14] Lopez-Ramirez F, Polyakov A, Efimov D, Perruquetti W. Finitetime and fixed-time observer design: Implicit Lyapunov function approach. Automatica 2018; 87(1): 52-60.

[15] Nakamura H, Yamashita Y, Nishitani H. Smooth Lyapunov functions for homogeneous differential inclusions. In: Proceedings of the 41st SICE Annual Conference. ; 2002: 1974-1979.

[16] Boiko I. Non-parametric Tuning of PID Controllers. London: Springer-Verlag . 2013.

[17] Sontag E. Nonlinear and Optimal Control Theory. Berlin: SpringerVerlag . 2007.

[18] Utkin V. Sliding Modes in Control Optimization. Berlin: SpringerVerlag . 1992.

[19] Husch L. Topological Characterization Of The Dilation and The Translation In Frechet Spaces. Mathematical Annals 1970; 190: 15 .

[20] Polyakov A. Generalized Homogeneity in Systems and Control. Springer . 2020.

[21] Bacciotti A, Rosier L. Lyapunov Functions and Stability in Control Theory. Springer . 2001.

[22] Boyd S, Ghaoui E, Feron E, Balakrishnan V. Linear Matrix Inequalities in System and Control Theory. Philadelphia: SIAM . 1994. 